\title{
Investor's satisfaction in portfolio selection problem
}

\author{
Aleksandra Rutkowska ${ }^{1}$ \\ ${ }^{1}$ Poznan University of Economics
}

\begin{abstract}
Empirical studies show that individual investors do not always behave rationally and do not use standard investment portfolio selection tasks. In this paper we focus on investor choices and the basic elements affecting them. The paper presents optimization model based on a measure of investor satisfaction. The model is created on the basis of surveys conducted among Polish individual investors. The fuzzy sets are used to model preference and expectation of investors and the uncertain future return rate of portfolio are fuzzy variable.
\end{abstract}

Keywords: fuzzy variable, portfolio optimization, investor's satisfaction

\section{Introduction}

The standard theory of individual decision making is the expected-utility (EU) theory proposed by Baniel Benroulli [2]. EU constitutes a key building block of a vast range of economic theory. However empirical studies dating from early 1950s have revealed a variety of pattern in choice bahavior that are inconsisten with EU. Modern portfolio theory, which was introduced by Harry Markowitz in a 1952 [14], attempts to maximize portfolio expected return for a given amount of portfolio risk, or equivalently minimize the risk for a given level of expected return, by carefully choosing the proportions of various assets. However, since prospect theory [7], it is well known that that people make decisions based on the potential value of losses and gains rather than the expected value and evaluate these values using certain heuristics. In practice, the economic data available are not only random, but mainly imprecise often expressed in the form of belief, approximate expression and even linguistic. Research on risks and uncertainties resulted in the development of different tasks to optimize investment decisions, from the classic, based on the probability measure, through those based on rough set theory and since 1990 s based on the fuzzy sets theory. Early researchers employed possibility as the basic measure of the occurrence of a fuzzy event and most of them devoted themselves to extending Markowitz's meanvariance selection idea. However, possibility measure is not self-dual. Therefore, Huang [12] proposed that we should use the self-dual credibility as the basic measure of the occurrence of a fuzzy event and study the fuzzy portfolio selection problems. The review of portfolio optimization using credibility theory can be found in [13]. Continuing the descriptive models' discussion, based on the results of the individual investors' surveys, the author proposes new portfolio selection model. The model uses the measure of satisfaction as a goal function. The paper is organized as follows. First, the background of project including conducted survey and satisfaction concepts is presented, then short preliminaries including fuzzy variable and fuzzy similarity measure. Next the investor's satisfaction model is introduced with example. The presented model combines normative and descriptive approach, therefore, the next subsection presents the results of empirical tests of the model. The last section summaries the paper.

\section{Project background}

Empirical studies, carried out in the eighties of the former century, shown the people choices are strongly influenced on the one hand by loss aversion and on the other hand by regret feeling. The loss aversion, first demonstrated by Amos Tversky and Daniel Kahneman [7], refers to people's tendency to strongly prefer avoiding losses to acquiring gains. While regret, presented by Loomes and Sudgen [11] and Bell [1], concerns the negative emotion experienced when learning that an alternative action would have resulted in a more successful outcome.

Recent surveys of individual investors in the world show them as a group of goals-oriented people. According Global Survey of Individual Investors $2014^{1}$, which were conducted across 14 countries, investors are also starting to look at risk and performance in more personal ways, which will help them better understand their goals and overall tolerance levels. A significant majority (63\%), for example, worries more about the risk of not achieving their own specific investment goals than not beating the broad market, two-thirds of investors $(67 \%)$ are willing to set a target return independent of the broad market and three-quarters (76\%) say they would be content to achieve their goals even if

\footnotetext{
${ }^{1} 2014$ Global Survey of Individual Investors http //www.fundresearch.de/sites/default/files/partnercenter/ Natixis/News/2014/NGAM_2014_Individual_Investor_ Survey_Full_Report.pdf
} 
it meant under-performing the market. A large majority $(73 \%)$ are confident their portfolios are based on personal goals and benchmarks.

In order to supplement the information from this survey we have prepared a questionnaire about portfolio choices, focused on the decision criteria and preferences. The survey was conducted among Polish individual investors.

\subsection{The individual investors survey}

The main sources of knowledge about individual investors in Poland are Warsaw Stock Exchange's (further: WSE) reports (limited mainly to study participation of different groups of investors in turnover on the Warsaw Stock Exchange) and the National Individual Investors' Survey (further: NIIS). The NIIS has been conducted annually since 2002 by the Polish Association of Individual Investors (furhter: AII). The association is the largest organization uniting stock investors in Poland, which has been operating since 1999 and belongs to the World Federation of Investors (WFIC). Its business activity is focused on education, analysis and protection of investor rights. In 2014, the NIIS covered 7023 questionnaires completed by investors from across Poland ( $15.6 \%$ of the respondents were members of AII). The results show a wide investor characteristics of their demographics, the portfolio information, motivations to invest, sources of information to opinions about the market. In order to supplement the information from this survey the author has prepared a questionnaire about the choice of the portfolio - the criteria and preferences. The survey was also conducted via the AII during the period from July 2013 to September of 2013. The survey consists of 14 closed questions. The questions concerning the investment decision: the applied analysis, risk assessment methods and the expected value. Other group questions were questions assessing the usefulness of various types of information and levels of satisfaction in comparison to the results of the investment.

According to NIIS: polish statistical investor is a young man aged approx. 38 years, living in a large city, having higher education, a good job. He wants to increase savings on the stock market and the investment is treated as an additional source revenue. Polish investor invests an average of 7 years, has up to 7 companies in the portfolio, and the value of his portfolio is approx. 30 thousand zlotys. More about the NIIS results can be found http://www.sii.org.pl/.

From the point of view of the described model, the most important information reaching out from the survey was that the investment goal is described by the expected rate of return and its values determined in a fuzzy way, usually with a range. Over $60 \%$ of respondents admitted that their expectations for returns are variable depend on current conditions and sentiment in the market, nearly $14 \%$ makes them dependent on interest rates for deposits and other instruments, and less than $9 \%$ of acquired experience. Only $4.5 \%$ of people have some constant expectations, and $9 \%$ is not able to assess what influences their expectations. Only $6 \%$ of respondents say that their expectations are a crisp number, the half express expectations by infinite range, specifying the minimum acceptable rate of return, and $42 \%$ by finite interval, not including the profits above a certain improbable level. When assessing the investment, more than $39 \%$ compared result with the planned profit, over $25 \%$ with interest rate of deposits / bonds, and one in five person with a score of selected index. Only 10\% of investors compared achieved return rate with the best results in a given period, and only 5 people pointed to the one with be worst result. Over $75 \%$ people among those comparing the result with a chosen index, conditioned their expectated rate of return on the conditions and mood on the market. Analogically, among people conditioning the rate of return on experience, over $65 \%$ compared the results with the expected profit. Over $60 \%$ of people conditioning their expectations on the interest rate and other instruments, compared the results with them. However, among investors, who comparing results with the planned profit, more than half (57\%) addictive expected rate of return of moods and conditions on the market. There were no significant effect of age, experience, or education on how the respondents assessed the investment.

\subsection{Investor satisfaction}

In terms of investment and investor preferences an utility function is well-known measure. An utility function shows the relationship between utility and return (or wealth). The optimal investment problem consists in finding the portfolio strategy that maximizes the expected value of the utility function at some predetermined moment in the future. There has been an attempt to define a new model for portfolio optimization based on the conclusions of the survey results. The model assumes that the investor evaluates investments based on perceived satisfaction from it, which is not always equivalent to an expected return rate or an expected utility. In the case of good financial market conditions, satisfaction with low rates of return will be less than with the same rate of return obtained bear market. In addition, investing in assets more uncertain, which probably gives more return, will be more satisfying for gamblers, who are gains oriented and have lower risk aversion. The concept of satisfaction is rarely discussed in terms of investment, what is the basic concept of management in terms of customer satisfaction. Kotler [8] defined customer satisfaction as a person's feelings of pleasure or disappointment resulting from comparing a product perceived performance (or outcome) in relation to his or her expectations. The conducted survey of individual 
investor has shown that when it comes to investment, the situation is analogous, therefore the investor satisfaction was defined as a degree of similarity investments result (a priori the credibility of particular return rates) that its expectations. The review of approaches to quantifying similarity and compatibility within the framework of fuzzy set theory can be found in [4].To measure the degree of similarity Tversky fuzzy index is choosed, whereby the level of satisfaction may be subject not only expectations but also from aversion to losses or gains orientation. The presented measure of satisfaction can be considered a special type of utility function, but the main difference is that the measure of satisfaction takes into account the expectations of investors and is based on the human method of comparing two sets.

\section{Preliminaries}

In this paper fuzzy variables are used to express uncertainty of future asset returns. This section brief reviews some basic concepts of fuzzy variable and similarity measures within the framework of credibility theory. In order to measure a fuzzy event, Zadeh proposed the concept of possibility measure in 1978 [16]. Then Didier Dubois and Henri Prade further contributed to its development. The overview of possibility theory, emphasizing its historical roots and its recent developments can be found in [5]. In order to define a self-dual measure, Liu and Liu gave the concept of credibility measure in 2002 [10]. Credibility theory and details of fuzzy variable can be found in [9].

\subsection{Fuzzy variable}

Credibility measure was defined by Liu and Liu in [10] as follows. Let $\Theta$ be a nonempty set, and $P$ the power of set $\Theta$. Each element in $P$ is called an event and $C r$ a credibility measure, which indicates the credibility that an event will occur.

Credibility is an arithmetic average of well-know possibility and necessity measures and satisfies the following four axioms:

1. normality: $\operatorname{Cr}\{\Theta\}=1$,

2. monotonicity: $\operatorname{Cr}\{A\} \leq \operatorname{Cr}\{B\}$ whenever $A \subset B$,

3. self-duality: $\operatorname{Cr}\{A\}+C r\{\bar{A}\}=1$, for any event $A$,

4. maximality: $\operatorname{Cr}\left\{\bigcup_{i} A_{i}\right\}=\sup _{i} \operatorname{Cr}\left\{A_{i}\right\}$ for any event $A_{i}$ with $\sup _{i} \operatorname{Cr}\left\{A_{i}\right\}<0.5$.

A fuzzy variable $\xi$ is defined in [10] as a function from a credibility space $(\Theta, P, C r)$ to the set of real numbers.

Suppose $\xi$ is a fuzzy variable with membership function $\mu$ and $x$ is a real number. For any $B \in \Re$, the credibility measure of event $\{\xi \in B\}$ was defined by
Liu and Liu [10] as:

$$
\begin{gathered}
C r\{\theta \in \Theta: \xi(\theta) \in B\}=\frac{1}{2}\left(\sup _{x \in B} \mu(x)+\right. \\
\left.1-\sup _{x \in B^{c}} \mu(x)\right), \forall x \in \Re
\end{gathered}
$$

Each fuzzy variable has a fuzzy membership function $\mu$-determines the possibility that the variable adopt a specific value:

$$
\mu(x)=\min (2 \operatorname{Cr}\{\theta \in \Theta: \xi(\theta)=x\}, 1)
$$

Fuzzy variable is called triangular if it has a triangular membership function, defined the following function and abbreviated written as $\xi=(a, b, c)$ :

$$
\mu(x)=\left\{\begin{array}{cc}
\frac{x-a}{b-a} & \text { for } a \leq x<b \\
1 & \text { for } x=b \\
\frac{c-x}{c-b} & \text { for } x \leq c \\
0 & \text { for others }
\end{array}\right.
$$

\subsection{Similarity measure}

Assessing the degree to which two objects or two concepts are similar is a fundamental component of human reasoning. Similarity assessment process based on a set of features of two objects was described by Tversky in [15]. In his axiomatic theory of similarity, comparison is achieved through a measure determining the common and the different features. This paper is based on concepts developed by Tversky and presented in fuzzy environment by Bouchon-Meunier et al. in [3].

For any set $\Omega$ of elements, let $F(\Omega)$ denote the set of fuzzy subsets of $\Omega$, and $\mu_{A}$ the membership function of any description $A$ in $F(\Omega)$. Comparison of the two fuzzy sets A and B defined in the same space takes into account elements of $\Omega$, which belong to the sets at least in part and depending on the situation also the elements which belong to $\mathrm{A}$ and not belonging to the set $\mathrm{B}$ and / or to set $\mathrm{B}$ and not to set A. T-norm can be used to make the intersection of two fuzzy sets. The most common method of calculating membership function of the product of the collection is the operator MIN, so:

$$
\mu_{A \cap B}(x)=\min \left(\mu_{A}(x), \mu_{B}(x)\right), \forall x \in \Omega .
$$

Via the difference $A-B$ we understand:

$$
A-B=A \cap \bar{B}, \text { where } \mu_{\bar{B}}=1-\mu_{B} .
$$

In [3] the fuzzy set measure $M$ has following definition. A fuzzy set measure $\mathrm{M}$ is a mapping: $F(\Omega) \rightarrow \Re^{+}$such that, for every $A$ and $B$ in $F(\Omega)$ :

1. $M(\oslash)=0$,

2. if $B \subseteq A$, then $M(B) \leq M(A)$.

The fuzzy set measure evaluates the weight of the elements of the universe characterized by a fuzzy set. The following fuzzy set measure is used in measure of comparison in infinite case:

$$
M(A)=\int_{\Omega} \mu_{A}(x) d x
$$


A M-measure of comparison on $\Omega$ is a mapping $S: F(\Omega) \times F(\Omega) \rightarrow[0,1]$ such that

$$
S(A, B)=F_{s}(M(A \cap B), M(B-A), M(A-B)),
$$

for a given mapping $F_{s}: \Re^{+} \times \Re^{+} \times \Re^{+} \rightarrow[0,1]$ and a fuzzy measure $M$ on $\Omega$.

A M-measure of similitude on $\Omega$ is an M-measure of comparison $S$ satisfying:

1. $F_{s}(u, v, w)$ is non-decreasing in $u$,

2. non-increasing in $v$ and $w$.

In [3] there were specified three groups of similarity measures: measures of satisfiability, measures of inclusion, measures of resemblance. The first group consider compatibility as an object with a reference object or class, the second one also concerns a situation with a reference object but measures whether the point common to $\mathrm{A}$ and $\mathrm{B}$ are important with regard to $\mathrm{A}$. The third one is used for a comparison between the descriptions of two objects of the same level.

A M-measure of satisfiability on $\Omega$ is an M-measure of similitude S such that:

1. $F_{s}(0, v, w)=0$ whatever $v$ and $w$ may be,

2. $F_{s}(u, v, w)$ is independent of $w$,

3. $F_{s}(u, 0,)=$.1 whatever $u \neq 0$ may be.

A M-measure of inclusion $\mathrm{S}$ on $\Omega$ is a M-measure of similitude such that:

1. $F_{s}(0, v, w)=0$ whatever $v$ and $w$ may be,

2. $F_{s}(u, v, w)$ is independent of $v$,

3 . is reflexive.

A M-measure of resemblance on $\Omega$ is a M-measure of similitude $\mathrm{S}$ which satisfies properties of:

1. reflexivity: $S(A, A)=1$,

2. symmetry: $S(A, B)=S(B, A)$.

The above definition is compatible with Tversky index in the contrast model presented in [15], as (1) for objects $\mathrm{x}$ with features set $\mathrm{X}$ and $\mathrm{y}$ with features set $\mathrm{Y}$.

$$
S_{x, y}=\frac{f(X \cap Y)}{f(X \cap Y)+\alpha f(X-Y)+\beta f(Y-X)},
$$

where $\alpha, \beta \geq 0,0 \leq S \leq 1$. Note that if $f$ is a fuzzy set measure the (1) is an f-measure of similitude. Using (3.2), (3.2), (3.2), (1) to evaluate similarity of two fuzzy sets $\mathrm{A}$ and $\mathrm{B}$ we use the below function:

$$
\begin{aligned}
& S=\int_{\Omega} \min \left(\mu_{A}, \mu_{B}\right) /\left(\int_{\Omega} \min \left(\mu_{A}, \mu_{B}\right)+\right. \\
& \left.\alpha \int_{\Omega} \min \left(\mu_{A}, \mu_{\bar{B}}\right)+\beta \int_{\Omega} \min \left(\mu_{\bar{A}}, \mu_{B}\right)\right) .
\end{aligned}
$$

\section{Investor's maximum satisfaction model}

In this section, the main concept of investor's satisfaction model is presented, then it is collected into a portfolio optimization model. Let assume:
- $\Theta$ is the set of all elementary market states $\theta$;

- $P$ is a power set of $\Theta$;

- $\xi:(\Theta, P, C r) \rightarrow \Re$ - fuzzy return rate, which determines the future value of return;

- $\mu: \Re \rightarrow[0,1]$ - membership function of fuzzy variable, assigns each value a possibility of occurance;

- $C r: P \rightarrow[0,1]$ - credibility function, determines the credibility of the event that the variable takes the value from a given range.

- $\gamma$ is a fuzzy set corresponding investor's expectations.

Investment objectives are set out in principle by a target rate of return on investment in a given time horizon, but its value is not a crips value. This means that the investor assumes a profit of $r$, it will be fully satisfied to achieve it or make it higher, however getting a rate of return little lower, but higher than risk-free rate, will be partially satisfying. An assessment of preferences can be determined by a fuzzy number "at least r", with membership function (2) and will be marked in short $\gamma=\left(r_{0}, r\right)$.

$$
\gamma(x)=\left\{\begin{array}{cc}
0 & \text { for } x \leq r_{0} \\
\frac{x-r_{0}}{r-r_{0}} & \text { for } r_{0}<x \leq r \\
1 & \text { otherwise }
\end{array}\right.
$$

The level of investor's satisfaction is determined by the similarity of credibility of the fuzzy returns to the investor's expectations. To measure similarity we will use the Tversky index (1). The satisfaction function is (3).

$$
\begin{aligned}
& S=\int_{r_{\min }}^{r_{\max }} \min \{C r(\theta \in \Theta: \xi(\theta)=r), \gamma\} / \\
& \left(\int_{r_{\max }}^{r_{\max }} \min \{C r(\theta \in \Theta: \xi(\theta)=r), \gamma\}+\right. \\
& \alpha \int_{r_{\min }}^{r_{\max }} \min \{C r(\theta \in \Theta: \xi(\theta)=r), \bar{\gamma}\}+ \\
& \left.\beta \int_{r_{\text {min }}}^{r_{\text {max }}} \min \{\overline{C r(\theta \in \Theta: \xi(\theta)=r)}, \gamma\}\right) \text {, }
\end{aligned}
$$

where

$$
\begin{gathered}
r_{\max }=\max \{\min \{r: \gamma(r)=1\}, \\
\max \{r: C r(\theta \in \Theta: \xi(\theta)=r) \geq 0\}\} \\
r_{\min }=\min \{\max \{r: \gamma(r)=0\}, \\
\min \{r: C r(\theta \in \Theta: \xi(\theta)=r) \geq 0\}\}
\end{gathered}
$$

Parameter $\alpha$ is interpreted as risk aversion parameter and $\beta$ as parameter of regret. Due to interpretation of the parameters: $\alpha$ and $\beta$, the parameters will be imposed an additional condition that: $\alpha+\beta=1$. It is worth noting that:

- if $\alpha=0$ and $\beta=1$, the (3) is a M-measure of satisfiability,

- if $\alpha=1$ and $\beta=0$, the (3) is a M-measure of inclusion,

- if $\alpha=0.5$ and $\beta=0.5$, the (3) is a M-measure of resemblance.

Each specific portfolio has the credibility function, which can be interpreted as the credibility of occurrence of the particular return rates, $\mathrm{Cr}$. In the case of portfolio selection let denote: 


\begin{tabular}{|c|c|c|c|}
\hline$\mu$ & $\alpha=0, \beta=1$ & $\alpha=0.5, \beta=0.5$ & $\alpha=1, \beta=0$ \\
\hline $\mathrm{A}(-2,0,3)$ & 0.1047 & 0.1469 & 0.2466 \\
$\mathrm{~B}(-6,-2,6)$ & 0.1988 & 0.2111 & 0.2249 \\
$\mathrm{C}(-1,0.08,2)$ & 0.0607 & 0.0962 & 0.2317 \\
\hline
\end{tabular}

Table 1: Satisfaction level for example variables with different levels of risk aversion and regret parameter

- $\xi_{i}$ - the $i$-th security fuzzy returns,

- $x_{i}$ - the investment proportions in the $i$-th securities, $i=1,2,3, \ldots, n$, respectively,

- $\operatorname{Cr}\left(\theta \in \Theta: \sum x_{i} \xi_{i}(\theta)=r\right) \cap \gamma$ - the level on which the portfolio meets the expectations,

- $\operatorname{Cr}\left(\theta \in \Theta: \sum x_{i} \xi_{i}(\theta)=r\right) \cap \bar{\gamma}$ - the level on which the portfolio does not meet the expectations,

- $\operatorname{Cr}\left(\theta \in \Theta: \sum x_{i} \xi_{i}(\theta)=r\right) \cap \gamma$ - the level on which others portfolios could meet the expectations, can be understood as opportunities cost level.

If the investor has a high risk aversion and a 0 regret parameter, his satisfaction level will be directly proportional to met expectations, and inversely proportional to the level of credibility of event realization returns rate below expectations and independent of unfulfilled opportunities. If the investor is characterized by a high rate of regret and very low risk aversion, his satisfaction level will be directly proportional to met expectations, and inversely proportional to opportunities cost.

Every investor has the same objective - the satisfaction maximization. So the investor's maximum satisfaction portfolio selection can be presented as following mathematical programming task (4).

$$
\begin{gathered}
\max S\left(C r\left(\theta \in \Theta: \sum x_{i} \xi_{i}(\theta)=r\right), \gamma\right) \\
\text { subject to: } \\
\sum_{i=1}^{n} x_{i}=1 \\
\forall_{i} x_{i} \geq 0
\end{gathered}
$$

\subsection{Example}

The example shows choice based on satisfaction level form 3 option: A, B and C. The investor's expectations is return at least $5(\gamma=(0,5))$. The options are following: A with membership function $\mathrm{A}=(-2,0,3), \mathrm{B}=(-6,-2,6), \mathrm{C}=(-1,0.08,2)$, see fig. 1 . The satisfaction level for investor with different risk aversion $(\alpha)$ and regret $(\beta)$ parameters are shown in table 1.

The investor with high regret parameters $(\alpha=0$ and beta $=1$ ), following the principle of maximum satisfaction, should be decided on the choice of B. Since this is the only one that gives credibility of return closer and higher than 5 . The investor with no regret parameters and maximum risk aversion $(\alpha=1$, beta $=0)$ should choose option $\mathrm{A}$ and choice $\mathrm{B}$ is the least satisfactory for him.

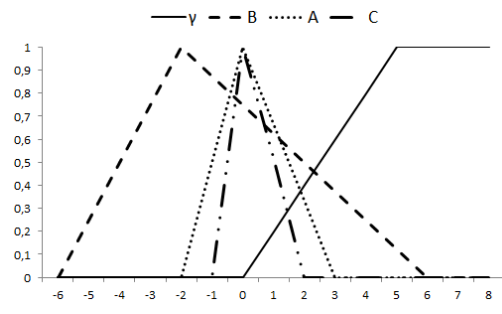

Figure 1: The example: membership functions and expectation

\begin{tabular}{|r|c|c|c|c|c|c|c|}
\hline period & A1 & A2 & A3 & A4 & A5 & A6 & A7 \\
\hline S1 & $-0,0877$ & 0,0768 & $-0,0617$ & 0,0149 & 0,1452 & 0,1096 & $-0,0218$ \\
S2 & $-0,0560$ & 0,1196 & $-0,0564$ & $-0,0169$ & 0,1050 & 0,1065 & $-0,0335$ \\
S3 & $-0,0584$ & 0,1279 & $-0,0528$ & $-0,0153$ & 0,0950 & 0,1070 & $-0,0302$ \\
WIG20 & $-0,0611$ & 0,0874 & $-0,0706$ & $-0,0177$ & 0,0534 & 0,0752 & $-0,0278$ \\
\hline
\end{tabular}

Table 2: Portfolios return rate in the test periods of study A

\subsection{The study}

The investors satisfaction model was tested on empirical data and the results compared with those of other fuzzy models known in the literature (for review of the models please see [6]). The empirical study is conducted on daily data of companies listed on the WSE during the the period from July 2008 to July 2013. The study selected companies in the index WIG20 (a capitalization-weighted stock market index of the twenty largest companies on the WSE), because, as statistics show, those companies are the preferred choice of individual investors - their share in the turnover of this group of investors in 2012 was almost $80 \%$. Empirical study was conducted on the following test periods:

- A: monthly, preceded by a quarterly period of observation of the market,

- B: quarterly, preceded by a semi-annual period of observation,

- C: half-yearly, preceded by a one-year period of observation,

- D: yearly, preceded by a 1,5 year period of observation data.

Thus, in case of $\mathrm{A}$, it will be 7 periods, and in case of B-D, 5 periods each.

During periods of longer length (studies C and D) an average of all the portfolios acquired results below WIG20. The best results (above the benchmark - WIG20) were obtained in the study of portfolios encompassing the shortest period (Study A). This

\begin{tabular}{|r|c|c|c|c|c|}
\hline & B1 & B2 & B3 & B4 & B5 \\
\hline S1 & 0,4524 & 0,0684 & 0,0578 & $-0,5171$ & $-0,1117$ \\
S2 & 0,4650 & 0,0599 & 0,0607 & $-0,5604$ & $-0,1242$ \\
S3 & 0,4507 & 0,0588 & 0,0630 & $-0,5648$ & $-0,1245$ \\
WIG20 & 0,3397 & 0,0307 & 0,0369 & $-0,0651$ & $-0,0427$ \\
\hline
\end{tabular}

Table 3: Portfolios return rate in the test periods of study B 
situation may be caused by an elongated data analysis period, what may provoke wrongly determined fuzzy return rates. In the test periods portfolios designated according to the criterion of satisfaction for the high risk aversion parameter shows lower returns amplitude than these for high regret parameters. There were no significant differences in the rates of return obtained from the test period compared to the results of tasks to maximize the satisfaction of the tasks that use fuzzy criteria for maximizing profit while reducing risk and minimizing risk at a given level of profit.

\section{Summary}

The paper presents new concept of portfolio selection. The author proposes single-criterion optimization task. The maximum investor satisfaction model makes the level of satisfaction dependant on the similarity of the result to the expectations, as well as the individual characteristics of investors their approach to risk and alternative choices. The next step of the research plan is to investigate the relationship between risk aversion and regret parameter and replace the values of risk aversion and regret with linguistic values.

\section{Acknowledgements}

The study has been prepared as a part of the research project of the National Science Center 2013/09/N/HS4/03761 using Infrastructures PLGrid.

\section{References}

[1] D.E. Bell, Regret in decision making under uncertainty, Operations research, 30(5):961-981, 1982.

[2] D. Bernoulli, Exposition of a New Theory on the Measurement of Risk, Econometrica, 22, 23-36, 1954, (1738 reprint).

[3] B. Bouchon-Meunier, M. Rifqi, and S. Bothorel, Towards general measures of comparison of objects, Fuzzy Sets Systems, 84(2):143-153, Elsevier North-Holland, 1996.

[4] V. V. Cross and T. A. Sudkamp, Similarity and Compatibility in Fuzzy Set Theory: Assessment and Applications, Studies in Fuzziness and Soft Computing, Physica-Verlag Heidelberg, 2002.

[5] D. Dubois and H. Prade, Possibility Theory and its Applications: Where Do we Stand?, 2011.

[6] X. Huang Portfolio Analysis - From Probabilistic to Credibilistic and Uncertain Approaches, Studies in Fuzziness and Soft Computing 250, Springer, 2010.

[7] D. Kahneman and A. Tversky, Choices, Values, and Frames, American Psychologist, 39(4):341350, 1984.

[8] P. Kotler, Marketing Management, 10 ed., Prentice-Hall, 2000.
[9] B. Liu, Uncertainty Theory, Studies in Fuzziness and Soft Computing, Springer Berlin Heidelberg, 2007.

[10] B. Liu and Y-K Liu, Expected value of fuzzy variable and fuzzy expected value models, Fuzzy Systems, IEEE Transactions on, 4:445-450, 2002.

[11] G. Loomes and R. Sugden, Regret theory: An alternative theory of rational choice under uncertainty, Economic Journal, 92(4):805-24, 1982.

[12] X. Huang, Risk Curve and Fuzzy Portfolio Selection, Computers and Mathematics with Applications, 55, 1102-1112, 2008.

[13] X. Huang, Portfolio Analysis From Probabilistic to Credibilistic and Uncertain Approaches, Studies in Fuzziness and Soft Computing, Springer, 2010.

[14] H.M. Markowitz, Portfolio Selection, The Journal of Finance, 7 (1): 77-91, 1952.

[15] A. Tversky, Features of Similarity, Psychological Reviews, 84(4): 327-352, American Psychological Association, 1977.

[16] L. A. Zadeh, Fuzzy sets as a basis for a theory of possibility, Fuzzy Sets and Systems, 1: 3-28. 